\title{
AFINIDADES EM REDES SOCIAIS E SUA RELAÇÃO COM DISPOSIÇÃO EM COLABORAR: ANÁLISE DE CASO EM UMẢ PEQUENA EMPRESA
}

\section{AFFINITIES IN SOCIAL NETWORKS AND ITS RELATION WITH COLLABORATIVE BEHAVIOR: CASE ANALYSIS IN A SMALL COMPANY}

\begin{abstract}
Resumo
O objetivo central desta pesquisa foi identificar se os indivíduos que recebem maior indicação de afinidade pelos membros da rede social em que estão inseridos na empresa são aqueles considerados possuidores de maior participação colaborativa pelos gestores. $O$ estudo foi realizado com 17 funcionários do setor produtivo de uma pequena empresa metalúrgica localizada em São Caetano do Sul - SP. Inicialmente, utilizou-se a técnica de Análise de Redes Sociais (SNA Social Network Analysis) através do software UCINET 6.0, considerando-se o critério de afinidade. Cada funcionário indicou um nível de afinidade que possui com cada um dos demais participantes da rede. A SNA permite diversas análises das relações entre os componentes da rede úteis para os gestores, e algumas delas são mostradas neste artigo. Porém, o foco principal do trabalho foi primeiramente identificar quais indivíduos (nós da rede) recebem maior número de manifestações de afinidade (variável afinidade). Numa segunda etapa, coletou-se a percepção dos gestores do setor com relação à disposição em colaborar de cada funcionário (variável disposição em colaborar). Finalmente, utilizando o software SPSS 15, realizou-se teste estatístico onde foi verificada a existência de forte correlação entre as duas varáveis. Assim, o estudo indicou que os funcionários que recebem maior número de manifestações de afinidade dos colegas são os que têm maior disposição em colaborar, segundo os gestores da empresa. Esta constatação indica que, se os gestores buscarem formas de estimular a inserção social dos funcionários, provavelmente haverá aumento nos níveis de participação colaborativa.
\end{abstract}

Palavras Chave: redes sociais; colaboração; afinidade.

\section{Introdução}

O cenário atual das organizações considera os funcionários como fundamentais para a organização e não mais como, somente, atores de produção. Nesse novo cenário, os funcionários tornaram-se peças fundamentais para a solução de problemas e, com isso, o patrimônio humano das 
organizações passou a ser visto como diferencial competitivo, e os indivíduos como os principais atores para formação de um ambiente propício ao desenvolvimento da organização. Por isso, é de suma importância que os gestores incentivem a disposição em colaborar dos funcionários no dia a dia da empresa, fazendo com que a organização se torne uma estrutura orgânica, em um ambiente colaborativo e participativo. Assim, as organizações podem se tornar mais dinâmicas, através de um fluxo de informações e conhecimentos que podem ser transmitidos horizontalmente.

A propagação das informações muitas vezes é feita por meio do conhecimento tácito, aquele que pertence ao capital intelectual da pessoa e apresenta dificuldade de mensuração em relação a sua origem e movimentação. Mais do que nunca, o conhecimento é hoje decisivo para o sucesso coorporativo e por isso o capital industrial vem sendo substituído cada vez mais por esse novo tipo de ativo, o capital intelectual. Portanto, o estudo das redes sociais é fundamental para a gestão do conhecimento organizacional, pois é através dessas redes que o conhecimento tácito é transmitido. Através de um mapeamento da rede social, pelo qual se podem mensurar os tipos de relações existentes, é possível ter conhecimento sobre a movimentação das informações e com isso a identificação do grau de inserção de cada indivíduo na rede.

O objetivo deste artigo foi verificar se os funcionários que foram identificados como sendo os possuidores de maior grau de inserção na rede social da empresa através do critério afinidade, são aqueles que, na percepção dos gestores, possuem maior disposição em colaborar. A colaboração, na dimensão social, tem um papel fundamental no desenvolvimento das interações que conduzem às diferentes formas de participação e criação de conhecimento, a partir da rede de relações entre os membros. Assim, este estudo tem relevância, à medida que busca verificar a correlação entre grau de afinidades e disposição em colaborar de funcionários. O melhor entendimento desta relação pode dar subsídios a ações que visam melhorar o nível de colaboração de funcionários, por meio de esfoços para incrementar as relações sociais existentes entre eles.

Para entendimento da forma de análise das redes sociais entre os funcionários dessa empresa, e se o grau de inserção dos indivíduos nesta rede está correlacionado com sua disposição em colaborar, será apresentado um referencial teórico sobre o assunto. Para a elaboração do instrumento de análise, será apresentado o modelo de Hatala (2006), que serviu de base para dar, através do software UCINET 6.0, subsídios para o levantamento da análise do objetivo pretendido. As análises de correlação entre as variáveis foram feitas utilizando o software SPSS 15 pelo qual foi identificada uma forte correlação entre as variáveis levantadas.

\section{Plataforma teórica}

Este capítulo tem como finalidade o embasamento teórico para a justificativa da pesquisa e da metodoligia utilizada. Está dividido em dois itens. Primeiramente, a teoria sobre redes sociais. 
Esta traz a teoria de introdução às redes, e de mapeamento e avaliação de redes sociais. Em seguida, uma discussão acerca da intensidade da inserção em uma rede social e a relação desta intensidade com a disposição em colaborar das pessoas desta rede, o que foi o principal foco desta pesquisa, aplicada a uma pequena empresa metarlúrgica de São Caetano do Sul - São Paulo.

\subsection{Redes sociais}

O objetivo deste item é o de posicionar o leitor acerca das teorias de redes utilizadas para este estudo. Primeiramente foi explorada a introdução às redes, onde é trazido os tipos de redes sociais. Em seguida é explorada a teoria acerca da metodologia de mapeamento e avaliação das redes sociais utilizadas para a elaboração da metodologia deste trabalho.

\subsubsection{Introdução às redes}

As redes podem ser entendidas como um espaço de atores no qual as fronteiras podem ou não possuir limites, representando um sistema de apoio ou um sistema físico constituído por um conjunto de participantes autônomos, que unem idéias e recursos em torno de valores e interesses compartilhados (MARTELETO, 2001). Assim, os valores e interesses de uma rede são constituídos pelas informações importantes para o ambiente organizacional.

Huysman e Wulf (2006) afirmam que a necessidade de gerir essas informações tem se mostrado importante para as organizações já que, os ativos intangíveis ganham maior valor na busca pela competitividade.

Em paralelo à evolução dos sistemas administrativos, surgem as redes informais que se orientam, naturalmente com o intuito de atender às necessidades de informações dos seus usuários (MARTELETO, 2001). Dessa forma, essas redes ou networks são representações da união de idéias e recursos em torno de valores e interesses compartilhados constituindo um sistema de apoio onde todos podem ser beneficiados.

Por esta razão torna-se necessário o desenvolvimento de ferramentas que incorporem a realidade informal das comunidades de compartilhamento de conhecimento e também que os gestores foquem no aspecto informal ao invés da estrutura formal da empresa. A busca dessas ferramentas, que tem o propósito de capturar a realidade informal das comunidades de compartilhamento de conhecimento, é ocasionada pela inovação, no entanto, Fleming e Marx (2006) afirmam que a inovação não é produto de apenas uma pessoa, mas sim produto de um trabalho árduo de pesquisa coletiva.

Neste contexto as comunidades, que são constituídas por grupos de pessoas que se reúnem para discutir determinado assunto, têm se mostrado uma importante ferramenta por possuírem uma característica informal e colaborativa. Portanto, a grande vantagem delas é o fato de se encontrarem, 
na prática e na troca de experiências, uma solução para o aprendizado e para a resolução de problemas em um tempo menor do que aconteceria normalmente (WENGER e SNYDER, 2001; HUYSMAN e WULF, 2006).

Existem algumas razões para que as comunidades se formem, mas uma delas é o fato de as pessoas compartilharem objetivos e trocarem conhecimentos por meio dessas, tornando a comunidade um benefício para a rede. Para poder aproveitar os benefícios oferecidos pelas redes informais os gestores devem focar em fomentar e desenvolver novas formas de avaliação. Por isso, o gerenciamento de comunidades informais é importante, pois acelera o desenvolvimento e a inovação (RIZOVA, 2002; BENGHOZI, 2006).

Apesar da importância e do grande interesse surgido em torno das redes informais, os gestores das empresas não possuem uma noção clara de como gerenciar esta estrutura e por esta razão acreditam em mitos quando precisam lidar com redes (CROSS; NOHRIA e PARKER, 2002). Um desses mitos, segundo os autores, descreve que "Nós não podemos fazer muito para socorrer as redes informais": Isto se deve a crença dos gestores de que essas redes dependem em grande parte dos relacionamentos pessoais, mas na realidade, os gestores podem ajudar através de uma mudança de estrutura organizacional, ou seja, analisando que pontos da estrutura atrapalham o fluxo de informação.

Em seu estudo Rizova (2002) mapeou cinco tipos de redes sociais, que podem coexistir ao mesmo tempo dentro de uma rede social, formadas por colaboradores, a saber:

a) Rede instrumental: constituída a partir do conteúdo trocado por profissionais para desenvolverem os seus trabalhos, tais como assuntos relacionados a transferências físicas, informações e recursos financeiros. Para o mapeamento desta rede os funcionários indicam quem primariamente eles procuram quando possuem dúvidas ou problemas;

b) Redes expressivas: são marcadas por relacionamentos de amizade e suporte social. Esses relacionamentos não são mapeados pela estrutura formal, regras e procedimentos porque não envolvem diretamente atitudes e comportamentos relacionados ao trabalho. Para mapeá-la, pergunta-se aos entrevistados: "com quem você se sente à vontade para discutir assuntos relacionados ao trabalho";

c) Redes de aconselhamento técnico: incluem canais aos quais engenheiros, técnicos ou profissionais conseguem aconselhamento técnico para assuntos que importam a organização, tais como gestão de projetos etc. Para o mapeamento desta rede pergunta-se às pessoas o seguinte: "quem você procurava quando precisa de uma pessoa com competência técnica e habilidades";

d) Redes de aconselhamento organizacional: pergunta aos entrevistados: "a quem elas recorrem quando precisam de uma pessoa com competência organizacional”; 
e) Redes de amizade: pergunta às pessoas: “com quais membros da rede você possui mais afinidade".

Estabelecendo um novo paradigma na pesquisa sobre a estrutura social, a análise de redes é feita através do conjunto de relações que os indivíduos estabelecem através de suas interações e necessidades uns com os outros. Portanto, uma rede não se reduz a uma simples soma de relações existentes e sim a uma forma de influência sobre cada relação que existe nessa estrutura. Dessa forma, as Redes de Amizades podem ser utilizadas para mapear a disposição em colaborar de cada funcionário.

\subsubsection{Mapeando e avaliando as redes sociais}

As teorias das redes sociais iniciaram-se no começo da década de 1930, com três diferentes grupos (psicologia, antropologia e matemáticos). Morenos (1934) criou os diagramas que, basicamente, representam o mapeamento dos relacionamentos entre os indivíduos e que são representados como pontos conectados por linhas (geometria dos relacionamentos interpessoais). Os diagramas foram produzidos para ajudar a identificar lideres de grupos, isolamentos, direção dos nós e reciprocidade entre os círculos de amizade (HATALA, 2006).

A ligação mais popular que é feita com a teoria das redes sociais, é o capital social, já que, este apresenta um foco sobre a importância dos relacionamentos que as pessoas possuem. Por esta razão, pode-se focar em analisar a densidade das redes, pois o foco deste tipo de análise é verificar quais atores da rede estão interconectados. Desta forma, poderemos entender quem se comunica com quem e o que eles fazem na rede. Conectar pessoas para compartilhar conhecimento nos traz um importante instrumento para podermos avaliar e melhorar esta comunicação.

Para análise das redes sociais baseada no capital social Huysman e Wulf (2006) propõem as seguintes dimensões:

a) Dimensão de habilidade cognitiva do capital social: o seu foco é a habilidade humana em se conectar, cognitivamente, com outro. Tendo o propósito de busca e entendimento sobre ao que se referem quando se comunicam, ou seja, se localizar no contexto do assunto no qual está sendo discutido. A grande habilidade cognitiva de compartilhamento se refere, em grande aspecto, ao conhecimento tácito. Este conhecimento de difícil articulação e que depende de metáforas ou do aprendizado, está relacionado com a intenção de que estes possam ser compartilhados;

b) Dimensão baseada na motivação de relacionamento: refere-se à questão para quem e quando o conhecimento é compartilhado. É baseada nos atributos característicos de um relacionamento social tais como: confiança, respeito mútuo e reciprocidade generalizada. Se as 
redes possuem altos scores de relacionamento baseado em motivação do capital social, implicará que os membros estão intrinsecamente motivados para compartilhar conhecimentos uns com os outros.

Ao examinar, através dessas dimensões, as formas como os indivíduos se comportam dentro do grupo podem-se definir os relacionamentos que existem, para encorajar a exploração da estrutura das redes, e a partir disso, analisar como os indivíduos trabalham juntos para atingirem uma melhora de desempenho.

Hatala (2006), utilizando a técnica Social Network Analysis, mede os relacionamentos na rede por meio de uma escala binária ou de valor. Essas medidas podem ser simplesmente indicadas por 0 ou $1(0$ = Não tem relacionamento ou $1=$ Possui relacionamento $)$, podendo ser útil quando o pesquisador estiver mapeando redes de grandes organizações. Se o pesquisador estiver por outro lado interessado em saber qual é a força desses relacionamentos, poderia utilizar uma escala de medidas de valores que avalia as redes numa escala de LIKERT ( $1=$ Fraco ou Inexiste e 5= Forte).

Através dessa análise, a densidade global pode ser medida quando todos os caminhos que levam de um nó específico a todos os outros nós da rede são identificados. Sendo que, conforme o autor, densidade é a medida pelo nível de conexões existentes entre os indivíduos que fazem parte da rede e representa o numero atual de ligações com a proporção do total de ligações possíveis que podem existir. $\mathrm{O}$ valor da densidade é medida numa escala que varia de 0 a 1 , sendo que 1 representa completa densidade da rede. Se, por exemplo, uma rede tiver uma densidade de medida de 0,55 , o atual número de laços presentes dentro da rede é 55\% dos laços possíveis. Uma baixa densidade da rede indica uma conexão pobre da rede entre os membros do grupo e podem impactar no fluxo de informações requerido para um nível aceitável.

Para apresentação dos dados das redes sociais pode ser utilizada duas diferentes maneiras: Matriz de Dados e Diagramas.

\section{a) Matriz de dados}

Permite a demonstração de uma forma matemática à transformação da informação através de métricas como: In-degree centrality; Out-degree centrality; Betweenness centrality; Closeness centrality; Densidade e Reciprocidade. Essas métricas serão bem detalhadas na Tabela 1: 
Tabela 1 - Métricas Básicas de Redes

\begin{tabular}{|c|c|c|}
\hline \multicolumn{3}{|c|}{ Métricas para um nó } \\
\hline Métrica & Descrição & Cálculo \\
\hline In-degree centrality & $\begin{array}{l}\text { É o número de setas que entram em um nó } \\
\text { em um dado tipo de rede. Fornece uma } \\
\text { perspectiva de centralidade local. }\end{array}$ & Somatório das setas que entram no nó. \\
\hline Out-degree centrality & $\begin{array}{l}\text { É o número de setas que saem de um nó em } \\
\text { um dado tipo de rede. Fornece uma } \\
\text { perspectiva de centralidade local. }\end{array}$ & Somatório das setas que saem do nó. \\
\hline Betweenness centrality & $\begin{array}{l}\text { É um indicador de quanto um nó particular } \\
\text { está entre os vários outros nós na rede }\end{array}$ & $\begin{array}{l}\text { Número de vezes que o nó aparece como } \\
\text { caminho entre todos os nós, dividido pelo } \\
\text { número de caminhos existentes entre todos os } \\
\text { nós. }\end{array}$ \\
\hline Closeness centrality & $\begin{array}{l}\text { É um indicador de quanto um nó está no } \\
\text { menor caminho entre outros vários nós da } \\
\text { rede. Fornece uma perspectiva de } \\
\text { centralidade global. }\end{array}$ & $\begin{array}{l}\text { Somatório da distância entre um determinado } \\
\text { nó para com todos os outros da rede. Esse } \\
\text { valor é normalizado em relação ao nó de } \\
\text { menor valor. }\end{array}$ \\
\hline \multicolumn{3}{|c|}{ Métricas para grupos } \\
\hline Métrica & Descrição & Cálculo \\
\hline Densidade & $\begin{array}{l}\text { É o número de conexões existentes dividido } \\
\text { pelo número de conexões possíveis. }\end{array}$ & $\begin{array}{l}\text { Número de conexões existentes dividido pelo } \\
\text { número de conexões possíveis. }\end{array}$ \\
\hline Reciprocidade & $\begin{array}{l}\text { Indica qual a proporção de conexões que tem } \\
\text { uma relação de reciprocidade. }\end{array}$ & $\begin{array}{l}\text { Número de conexões bidirecionais } \\
\text { (recíprocas) dividido pelo número de } \\
\text { conexões. }\end{array}$ \\
\hline
\end{tabular}

Fonte: Adaptado de Guimarães e Sousa (2005)

\section{b) Diagramas}

Fornece uma representação visual dos dados, ou seja, contemplá-la como uma rede de relacionamentos diretos ou indiretos (ilustrados como uma linha entre dois círculos), os indivíduos são representados por círculos e a ligação entre eles por linhas com setas indicando a direção do relacionamento (LEA et ali, 2006). Essa análise visual permite que se tenha uma visão da rede como um todo. Porém, para redes com muitos nós essa visão pode ficar confusa, devido à grande quantidade de nós e relacionamentos. Uma solução para essa questão é utilizar o recurso da análise de métricas básicas de redes, apresentadas no tópico anterior.

A partir desses métodos, pode-se fazer uma análise de uma rede social constituída informalmente em uma empresa de pequeno porte. Assim, o resultado poderá dar subsídios para os gestores de como direcionar uma atividade que exija qualquer tipo de relacionamento social entre os funcionários.

\subsection{Redes sociais e disposição em colaborar}

De maneira geral, pode-se dizer que o termo Rede tem sido utilizado eminentemente por dois atores específicos: pelos cientistas e teóricos na pesquisa científica, e pelos atores sociais para designar algum tipo de relação ou prática social. Essa área de conhecimento indica que a rede é o 
único padrão de organização comum a todos os sistemas vivos, que compreende e perdura até os dias atuais.

As redes guardam características que favorecem trabalhos cooperativos, participativos, democráticos, e que exijam necessidades básicas comuns às práticas e objetivos de uma organização. Dentro de uma estrutura vertical em uma organização, a rede favorece a integração horizontal de todos os participantes como uma verdadeira malha de intermináveis fios. Através de formas de organização de trabalho colaborativo, cooperativo e integrado entre indivíduos ou grupos, que possuem objetivos comuns em maior ou menor grau de composição, essas redes constituem um conjunto de nós interconectados (CASTELLS, 2003).

Nas empresas, as redes sociais têm se formado com os seguintes objetivos: favorecer a circulação e a troca de informações, o compartilhamento de experiências, a realização de ações em conjunto, o aprendizado coletivo e a inovação, a criação e o fortalecimento de laços de solidariedade entre os membros, a manutenção da participação em comunidade e a ampliação das práticas colaborativas. Stahal (2006) afirma que através dessas práticas de colaboração, a aprendizagem em rede se constitui como um processo dinâmico de envolvimento partilha e construção conjunta do novo conhecimento realizado em uma empresa. Os processos colaborativos promovem a interpretação de uma construção colaborativa da compreensão, a qual se desenvolve através da elaboração da representação conjunta de um grupo de aprendizagem em rede.

Brown e Duguid (2002) confirmam que esses processos são desenhados através do percurso da definição de objetivos e práticas comuns orientadas para a partilha de experiências, métodos e estratégias, os quais são os elementos organizadores da atividade e organização do grupo, representando, deste modo, a possibilidade de desenvolvimento das interações sociais. Ou seja, o desenvolvimento colaborativo, em rede, pode ser desenvolvido a partir dos laços entre os indivíduos e seus contextos de aprendizagem e conhecimento.

Nessa abordagem, o desenvolvimento da participação colaborativa, é relacionado à experiência da utilização individual e do grupo, evidenciando deste modo que a colaboração depende, em larga medida, das pessoas que o vivem e a atualizam. Esta perspectiva coloca em destaque as interações entre os membros do grupo e a sua função. Podendo ser no desenvolvimento da dimensão social, ou também como meio para a experiência partilhada dos cenários de aprendizagem e produção do conhecimento que caracterizam a perspectiva dinâmica do contexto que emerge da rede social (FIGUEIREDO e AFONSO, 2006).

Ainda segundo os autores, a colaboração, na dimensão social, tem um papel fundamental no desenvolvimento das interações que conduzem às diferentes formas de participação e criação de conhecimento, a partir da rede de relações entre os membros. Visto desta forma, as redes sociais influenciam diretamente à colaboração participativa na empresa. Assim, essa correlação entre as 
teorias abordadas servirão como base para a análise desta pesquisa, como já mencionada anteriormente.

\section{Metodologia}

Trata-se de uma pesquisa do tipo exploratória que será estruturada a partir de um levantamento de campo, Gil (2006), onde o objetivo foi verificar se os funcionários que foram identificados como sendo os possuidores de maior grau de inserção na rede social da empresa através do critério afinidade, são aqueles que, na percepção dos gestores, possuem maior disposição em colaborar. Para teste desta correlação, utilizou-se a rede social no setor produtivo de uma empresa de pequeno porte, localizada em São Caetano do Sul - SP, pertencente ao setor metalmecânico, que possui 17 funcionários neste setor, além de um coordenador e um gerente. Salientamos que os nomes utilizados neste artigo são os apelidos ou nomes reais, e que houve consentimento dos envolvidos para que seus nomes fossem diretamente citados.

Primeiramente, através da distribuição de uma folha para cada um dos 17 funcionários do setor produtivo da empresa com a questão abaixo, foi solicitado que atribuissem uma nota entre 1 e 5 para cada colega de trabalho, afim de identificar o grau de afinidade que o respondente possuia com os outros 16 colegas:

"Levando em conta o seu grau de afinidade com os colegas abaixo, que valor você atribui a cada uma dessas pessoas? (coloque 99 no seu nome)

$1=$ (muito baixa afinidade)

$2=$ (baixa afinidade)

$3=($ nem baixa, nem alta afinidade

$4=($ alta afinidade $)$

$5=$ (muito alta afinidade)"

Deve-se destacar que não se pode garantir que os respondentes possuíam a mesma interpretação do termo "afinidade", ao responder ao questionamento, pois trata-se de um conceito subjetivo. Apesar da possibilidade desta variação no entendimento, pode-se considerar que há um nível aceitável de homogeneidade, especialmente quando se consideram os valores das extremidades (muito baixa afinidade e muito alta afinidade), que representam alta intensidade do sentimento. Porém, é possível $\mathrm{Na}$ folha também continha uma tabela com o nome dos 17 funcionários na qual a pesquisa foi executada, e um espaço para que o pesquisado pudesse atribuir o grau de afinidade para cada colega. Após o retorno dos 17 questionários aplicados aos funcionários, foi utilizado o Excel 2007 para tabulação dos dados, onde se obteve uma matriz de afinidades com os devidos valores atribuídos, como serão mostrados posteriormente na Tabela 2.

Entretanto, conforme Hatala (2006), o formato binário é requerido para descrever e analisar as relações de afinidade para uma melhor visualização no software de análise de intensidade de relação. Portanto, foi necessário dicotomizar os dados em um formato binário, onde foram 
considerados somente os valores iguais a 5 como objetos de análise, ou seja, as relações de muito alta afinidade. Assim sendo, todos os valores entre 1 e 4 foram substituídos por 0 e o valor 5 foi substituído por 1, como serão mostrados posteriormente na Tabela 3. Optou-se por considerar apenas o valor 5 (muito alta afinidade) como ocorrência efetiva de afinidade pela razão mencionada anteriormente, de que o valor extremo na escala diminui a possibilidade de diferença de entendimento dos respondentes com relação ao significado do termo "afinidade".

Essa tabela de valores dicotomizados foi transportada para o Ucinet $6 \mathrm{com}$ a finalidade de obter o desenho do diagrama da rede social, que será representado pela Figura 1. Além do diagrama, foi possível também, através do software Ucinet 6, verificar as métricas básicas de rede, conforme será mostrado na Tabela 4, as quais suas definições já foram detalhadas na Tabela 1.

Os resultados obtidos e a análise desses resultados também serão apresentados na próxima seção (Resultados).

Em seguida, foi entregue uma folha com a solicitação abaixo ao coordenador de produção e ao gerente de produção para que, juntos, atribuíssem valores de 0 à 10 aos 17 funcionários do setor produtivo da empresa. Esses valores atribuídos objetivavam representar, de acordo com a percepção desses gestores, a disposição do funcionário em colaborar (tecnicamente ou não) no ambiente de trabalho com os colegas e com a empresa.

"Atribua uma nota entre 0 e 10 para cada funcionário relacionado abaixo, representando a disposição em colaborar (tecnicamente ou não) no ambiente de trabalho com os colegas e com a empresa, onde 0 significa nenhuma disposição em colaborar e 10 significava total disposição em colaborar".

Nessa mesma folha, também continha uma tabela com o nome dos 17 funcionários na qual a pesquisa foi realizada, e um espaço para que os gestores pudessem atribuir um valor referente à disposição em colaborar de cada um deles. Após o retorno dessa folha, foi utilizado o Excel 2007 para tabulação de dados, onde se obteve uma tabela que mostra qual a disposição em colaborar de cada um dos funcionários, de acordo com a percepção dos gestores, como será mostrada posteriormente na Tabela 5.

A informação sobre o nível de disposição em colaborar foi coletada apenas dos gestores da empresa, por se considerar que estes são os principais conhecedores desta característica dos funcionários. Também os demais funcionários poderiam emitir opinião sobre este aspecto, porém com menor propriedade. Por isso, não foram ouvidos sobre este tema, além do fato de já estarem respondendo sobre o aspecto afinidade.

Por fim, para verificação das correlações, os dados foram imputados no SPSS 15 para uma análise estatística da pesquisa, como será mostrado na Figura 2, onde a variável Afinidade representa o Indegree, ou seja, o número de vezes que os demais colegas identificaram ter muito 
alta afinidade com aquele funcionário, e a variável Disposição em Colaborar provém dos valores atribuídos pelos dois gestores. Além da verificação da correlação dessas duas variáveis, foi feita também a correlação entre Disposição em Colaborar com: Idade do Funcionário, Setor onde Trabalha, Tempo de Casa e Salário. Essas outras variáveis foram levantadas junto ao Gerente Administrativo da Empresa.

Os resultados obtidos e a análise desses resultados serão apresentados na próxima seção (Resultados).

\section{Resultados}

A Tabela 2 representa a tabulação dos dados obtidos através da solicitação para que cada um dos 17 funcionários atribuíssem valores de 1 a 5 para o grau de afinidade que possuíam com os demais colegas do setor.

Tabela 2 - Matriz de Afinidades

\begin{tabular}{l|c|c|c|c|c|c|c|c|c|c|c|c|c|c|c|c|c}
\hline & Alex & Alípio & Anderson & Cipó & Tonho & HarryPoter & Negão & Bruno & Toco & SeuJoão & Juba & Juca & Bombril & Nilson & Betão & Tiago & Wilson \\
\hline Alex & 0 & 4 & 5 & 5 & 4 & 4 & 5 & 4 & 5 & 5 & 5 & 5 & 4 & 1 & 4 & 4 & 4 \\
\hline Alípio & 3 & 0 & 2 & 3 & 4 & 3 & 2 & 3 & 4 & 3 & 2 & 3 & 3 & 3 & 4 & 4 & 4 \\
\hline Anderson & 4 & 3 & 0 & 4 & 3 & 2 & 4 & 3 & 4 & 3 & 5 & 2 & 3 & 3 & 3 & 3 & 4 \\
\hline Cipó & 3 & 5 & 3 & 0 & 3 & 3 & 5 & 5 & 5 & 3 & 3 & 5 & 5 & 3 & 5 & 5 & 3 \\
\hline Tonho & 2 & 5 & 2 & 2 & 0 & 2 & 4 & 3 & 5 & 2 & 2 & 5 & 2 & 4 & 5 & 4 & 4 \\
\hline HarryPoter & 3 & 3 & 3 & 4 & 4 & 0 & 2 & 4 & 4 & 4 & 4 & 3 & 4 & 3 & 4 & 4 & 3 \\
\hline Negão & 3 & 5 & 1 & 5 & 5 & 3 & 0 & 3 & 5 & 3 & 5 & 2 & 2 & 5 & 1 & 5 & 5 \\
\hline Bruno & 2 & 3 & 1 & 3 & 3 & 2 & 3 & 0 & 3 & 2 & 4 & 4 & 3 & 1 & 3 & 4 & 2 \\
\hline Toco & 3 & 5 & 2 & 4 & 4 & 3 & 4 & 3 & 0 & 4 & 4 & 4 & 3 & 4 & 4 & 5 & 5 \\
\hline SeuJoão & 4 & 5 & 3 & 3 & 5 & 1 & 2 & 2 & 4 & 0 & 5 & 1 & 5 & 5 & 2 & 2 & 2 \\
\hline Juba & 4 & 4 & 4 & 4 & 5 & 5 & 5 & 4 & 4 & 4 & 0 & 5 & 3 & 4 & 2 & 4 & 4 \\
\hline Juca & 4 & 4 & 3 & 4 & 5 & 1 & 4 & 4 & 4 & 2 & 4 & 0 & 2 & 3 & 4 & 4 & 3 \\
\hline Bombril & 4 & 4 & 3 & 5 & 3 & 5 & 3 & 4 & 4 & 5 & 5 & 3 & 0 & 3 & 4 & 4 & 3 \\
\hline Nilson & 2 & 2 & 2 & 5 & 5 & 3 & 5 & 2 & 2 & 2 & 5 & 5 & 2 & 0 & 5 & 2 & 5 \\
\hline Betão & 3 & 5 & 2 & 5 & 5 & 3 & 3 & 3 & 5 & 4 & 3 & 5 & 3 & 3 & 0 & 5 & 4 \\
\hline Tiago & 3 & 5 & 1 & 2 & 4 & 2 & 2 & 5 & 5 & 2 & 3 & 5 & 3 & 3 & 4 & 0 & 4 \\
\hline Wilson & 2 & 5 & 3 & 5 & 5 & 5 & 4 & 4 & 5 & 3 & 5 & 5 & 4 & 4 & 4 & 5 & 0 \\
\hline FOnt: PeSqunncy
\end{tabular}

Fonte: Pesquisa de campo

A Tabela 3 representa os resultados da Tabela 2, porém com os valores dicotomizados, ou seja, todos os valores entre 1 e 4 foram substituídos por 0 e o valor 5 foi substituído por 1 . 
Tabela 3 - Matriz de Afinidades - Dados Dicotomizados

\begin{tabular}{l|c|c|c|c|c|c|c|c|c|c|c|c|c|c|c|c|c}
\hline & Alex & Alípio & Anderson & Cipó & Tonho & HarryPoter & Negão & Bruno & Toco & SeuJoão & Juba & Juca & Bombril & Nilson & Betão & Tiago & Wilson \\
\hline Alex & 0 & 0 & 1 & 1 & 0 & 0 & 1 & 0 & 1 & 1 & 1 & 1 & 0 & 0 & 0 & 0 & 0 \\
\hline Alípio & 0 & 0 & 0 & 0 & 0 & 0 & 0 & 0 & 0 & 0 & 0 & 0 & 0 & 0 & 0 & 0 & 0 \\
\hline Anderson & 0 & 0 & 0 & 0 & 0 & 0 & 0 & 0 & 0 & 0 & 1 & 0 & 0 & 0 & 0 & 0 & 0 \\
\hline Cipó & 0 & 1 & 0 & 0 & 0 & 0 & 1 & 1 & 1 & 0 & 0 & 1 & 1 & 0 & 1 & 1 & 0 \\
\hline Tonho & 0 & 1 & 0 & 0 & 0 & 0 & 0 & 0 & 1 & 0 & 0 & 1 & 0 & 0 & 1 & 0 & 0 \\
\hline HarryPoter & 0 & 0 & 0 & 0 & 0 & 0 & 0 & 0 & 0 & 0 & 0 & 0 & 0 & 0 & 0 & 0 & 0 \\
\hline Negão & 0 & 1 & 0 & 1 & 1 & 0 & 0 & 0 & 1 & 0 & 1 & 0 & 0 & 1 & 0 & 1 & 1 \\
\hline Bruno & 0 & 0 & 0 & 0 & 0 & 0 & 0 & 0 & 0 & 0 & 0 & 0 & 0 & 0 & 0 & 0 & 0 \\
\hline Toco & 0 & 1 & 0 & 0 & 0 & 0 & 0 & 0 & 0 & 0 & 0 & 0 & 0 & 0 & 0 & 1 & 1 \\
\hline SeuJoão & 0 & 1 & 0 & 0 & 1 & 0 & 0 & 0 & 0 & 0 & 1 & 0 & 1 & 1 & 0 & 0 & 0 \\
\hline Juba & 0 & 0 & 0 & 0 & 1 & 1 & 1 & 0 & 0 & 0 & 0 & 1 & 0 & 0 & 0 & 0 & 0 \\
\hline Juca & 0 & 0 & 0 & 0 & 1 & 0 & 0 & 0 & 0 & 0 & 0 & 0 & 0 & 0 & 0 & 0 & 0 \\
\hline Bombril & 0 & 0 & 0 & 1 & 0 & 1 & 0 & 0 & 0 & 1 & 1 & 0 & 0 & 0 & 0 & 0 & 0 \\
\hline Nilson & 0 & 0 & 0 & 1 & 1 & 0 & 1 & 0 & 0 & 0 & 1 & 1 & 0 & 0 & 1 & 0 & 1 \\
\hline Betão & 0 & 1 & 0 & 1 & 1 & 0 & 0 & 0 & 1 & 0 & 0 & 1 & 0 & 0 & 0 & 1 & 0 \\
\hline Tiago & 0 & 1 & 0 & 0 & 0 & 0 & 0 & 1 & 1 & 0 & 0 & 1 & 0 & 0 & 0 & 0 & 0 \\
\hline Wilson & 0 & 1 & 0 & 1 & 1 & 1 & 0 & 0 & 1 & 0 & 1 & 1 & 0 & 0 & 0 & 1 & 0 \\
\hline Font & & & & & & & & & & & & &
\end{tabular}

Fonte: Pesquisa de campo

A Figura 1 é o desenho do diagrama da rede gerada através do Software Ucinet 6. Utilizando os dados dicotomizados da tabela anterior. As linhas azuis demonstram muito alta afinidade em apenas um sentido, e as linhas vermelhas demonstram muito alta afinidade bilateralmente.

Figura 1 - Diagrama de Muito Alta Afinidade

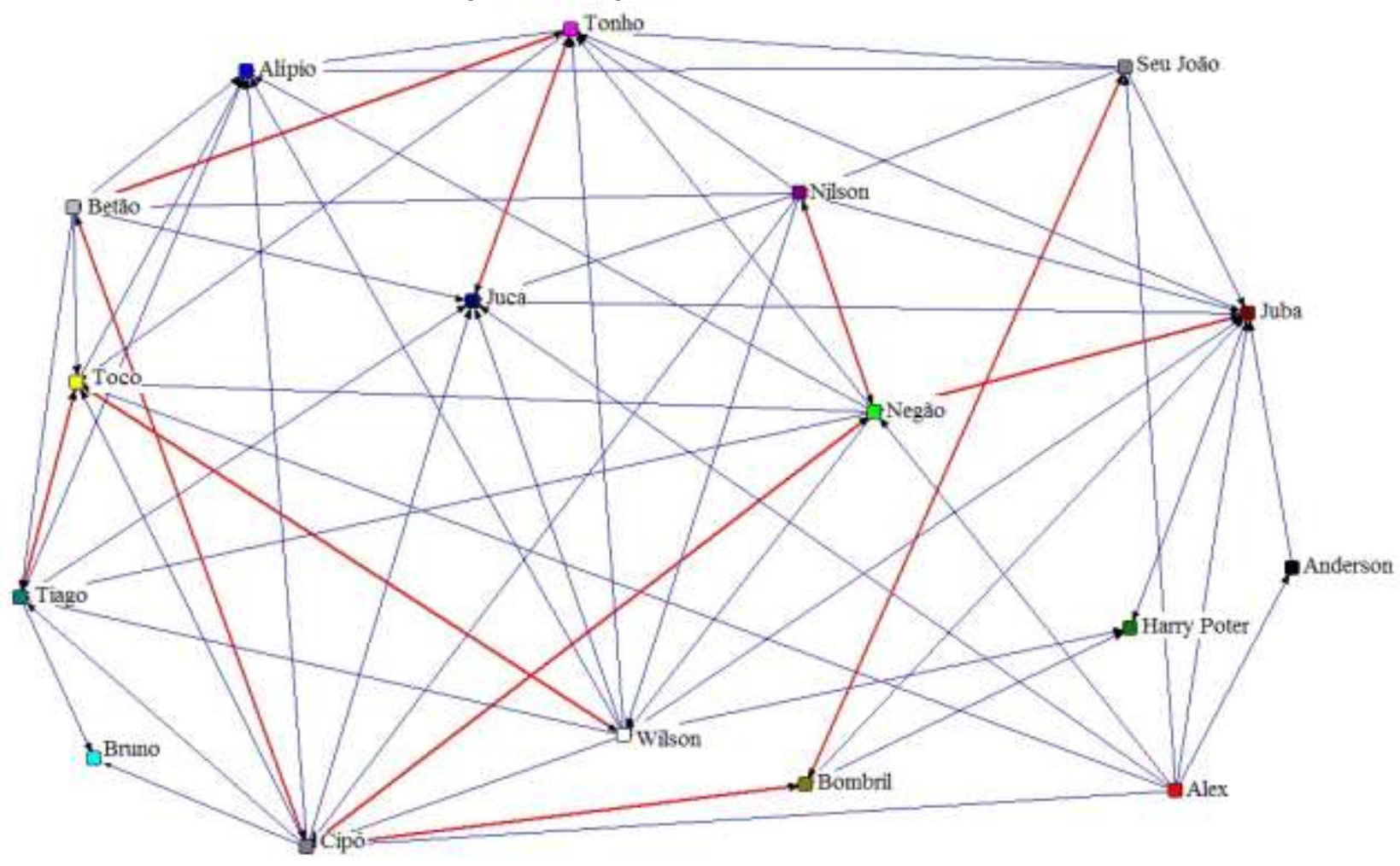

Fonte: Pesquisa de campo 
$\mathrm{Na}$ Tabela 4, gerada pelo software Ucinet 6 e adaptada a uma forma matricial, apresentam-se os valores métricos básicos da rede, já detalhados na Tabela 1.

Tabela 4 - Apresentação dos Valores Métricos Básicos da Rede

\begin{tabular}{c|c|c|c|c|c|c}
\hline ID & InDegree & OutDegree & Betweenness & Closeness & Densidade & Reciprocidade \\
\hline Alex & 0 & 7 & 8,1420 & 42 & & \\
\hline Alípio & 8 & 0 & 3,6300 & 42 & & \\
\hline Anderson & 1 & 1 & 0,0000 & 51 & & \\
\hline Cipó & 6 & 8 & 18,4070 & 38 & & \\
\hline Tonho & 7 & 4 & 3,8590 & 41 & & \\
\hline Harry Poter & 3 & 0 & 0,3300 & 48 & & \\
\hline Negão & 4 & 8 & 3,8880 & 40 & & $8,33 \%$ \\
\hline Bruno & 2 & 0 & 0,0000 & 52 & $88,24 \%$ & \\
\hline Toco & 7 & 3 & 2,5320 & 41 & & \\
\hline Seu João & 2 & 5 & 2,9830 & 44 & & \\
\hline Juba & 7 & 4 & 15,6440 & 40 & & \\
\hline Juca & 8 & 1 & 3,8460 & 41 & & \\
\hline Bombril & 2 & 4 & 2,1520 & 45 & & \\
\hline Nilson & 2 & 7 & 3,1150 & 41 & & \\
\hline Betão & 3 & 6 & 1,3970 & 44 & & \\
\hline Tiago & 5 & 4 & 5,2380 & 42 & & \\
\hline Wilson & 3 & 8 & 9,8330 & 39 & & \\
\hline Fon Pesquisa & 6 & & & & \\
\hline
\end{tabular}

Fonte: Pesquisa de campo

A partir dessa tabela pode-se obter algumas conclusões, como por exemplo que os funcionários Alípio e Juca são os que possuem maior índice de InDegree, ou seja, exitem 8 pessoas que identificaram possuir muito alta afinidade com esses, enquanto Alex não foi citado por nenhum funcionário, ou seja, nenhum dos colegas de trabalho possuem muito alta afinidade com esse. Esses dados podem mostrar ao gestor quais são os funcionários centrais da rede e quais são os isolados e que muitas vezes necessitam de um trabalho de inserção nesta rede social. $\mathrm{O}$ valor da densidade global da rede de $88,24 \%$ e a reciprocidade de $8,33 \%$ demonstra que apesar de a rede apresentar alta densidade, possui baiza reciprocidade.

A Tabela 5 representa a tabulação dos dados obtidos através da solicitação para que os gestores atribuíssem notas de 0 a 10 para cada um dos 17 funcionários que representam a disposição do funcionário em colaborar (tecnicamente ou não) no ambiente de trabalho com os colegas e com a empresa. 
Tabela 5 - Percepção do Coordenador de Produção e Gerente de Produção com Relação à Disposição em Colaborar dos Funcionários

\begin{tabular}{c|c}
\hline ID & Disposição em Colaborar \\
\hline Alex & 4,0 \\
\hline Alípio & 9,0 \\
\hline Anderson & 5,0 \\
\hline Cipó & 8,0 \\
\hline Tonho & 8,0 \\
\hline HarryPoter & 8,5 \\
\hline Negão & 8,0 \\
\hline Bruno & 7,0 \\
\hline Toco & 9,5 \\
\hline SeuJoão & 4,0 \\
\hline Juba & 8,0 \\
\hline Juca & 8,0 \\
\hline Bombril & 7,5 \\
\hline Nilson & 6,0 \\
\hline Betão & 7,0 \\
\hline Tiago & 9,0 \\
\hline Wilson & 9,0 \\
\hline
\end{tabular}

Fonte: Pesquisa de campo

A Figura 2 mostra os dados imputados no SPSS 15 para uma análise estatística da pesquisa.

Figura 2 - Dados no SPSS

\begin{tabular}{|c|c|c|c|c|c|c|c|}
\hline \multicolumn{8}{|c|}{ SLUP.sav [DataSet1] - SPSS Data Editor } \\
\hline File Eo & it View $\underline{D}$ a & ta Iransfo & rm Analyze Graphs Utilit & es. Wir & idow Help & & \\
\hline \multicolumn{8}{|c|}{ 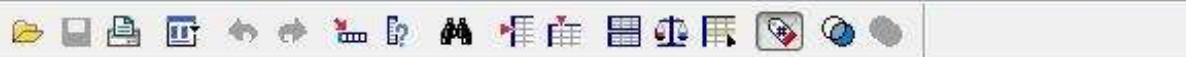 } \\
\hline \multicolumn{8}{|l|}{ 32: } \\
\hline & Nome & Afinidade & Disposição_em_colaborar & Idade & Setor & Tempo & Salário \\
\hline 1 & Alex & 0 & 4,0 & 22 & Bancada & 15 & 860,20 \\
\hline 2 & Alípio & 8 & 9,0 & 28 & $\mathrm{CNC}$ & 47 & 1163,8 \\
\hline 3 & Anderson & 1 & 5,0 & 18 & Bancada & 5 & 860,20 \\
\hline 4 & Cipó & 6 & 8,0 & 57 & Fresa & 6 & 1834,8 \\
\hline 5 & Tonho & 7 & 8,0 & 51 & Serra & 135 & 1036,2 \\
\hline 6 & HarryPoter & 3 & 8,5 & 53 & Torno & 14 & 1834,8 \\
\hline 7 & Negão & 4 & 8,0 & 53 & Bancada & 62 & 860,20 \\
\hline 8 & Bruno & 2 & 7,0 & 18 & $\mathrm{CNC}$ & 9 & 860,20 \\
\hline 9 & Toco & 7 & 9,5 & 36 & Expedição & 78 & 1524,6 \\
\hline 10 & SeuJoão & 2 & 4,0 & 52 & Bancada & 33 & 860,20 \\
\hline 11 & Juba & 7 & 8,0 & 55 & Torno & 136 & 1836,9 \\
\hline 12 & Juca & 8 & 8,0 & 26 & CNC & 29 & 1210,0 \\
\hline 13 & Bombril & 2 & 7,5 & 26 & Torno & 9 & 1163,8 \\
\hline 14 & Nilson & 2 & 6,0 & 38 & Bancada & 28 & 860,20 \\
\hline 15 & Betão & 3 & 7,0 & 51 & Fresa & 76 & 1834,8 \\
\hline 16 & Tiago & 5 & 9,0 & 24 & $\mathrm{CNC}$ & 69 & 1834,8 \\
\hline 17 & Wilson & 3 & 9,0 & 57 & Expedição & 67 & 1029,3 \\
\hline
\end{tabular}

Fonte: Pesquisa de campo

Os próximos quadros representam a análise estatística da pesquisa. O Quadro 1, extraído do SPSS 15, mostra o teste de aderência à curva normal das variáveis ordinais: 
Quadro 1 - Teste de Normalidade das Variáveis Ordinais

Tests of Normality

\begin{tabular}{|c|c|c|c|c|c|c|}
\hline & \multicolumn{3}{|c|}{ Kolmogorov-Smirnov ${ }^{9}$} & \multicolumn{3}{|c|}{ Shapiro-Wilk } \\
\hline & Statistic & df & Sig. & Statistic & df & Sig. \\
\hline $\begin{array}{l}\text { Afinidade - Dicotomizada } \\
\text { (Indegree) }\end{array}$ &, 196 & 17 &, 082 & ,913 & 17 &, 110 \\
\hline Disposição em Colaborar &, 230 & 17 &, 017 & 873 & 17 &, 025 \\
\hline Idade &, 256 & 17 &, 004 &, 851 & 17 &, 011 \\
\hline $\begin{array}{l}\text { Tempo de Empresa em } \\
\text { Meses }\end{array}$ &, 171 & 17 & $200^{*}$ &, 866 & 17 &, 019 \\
\hline Salário Mensal & ,208 & 17 &, 049 & .786 & 17 &, 001 \\
\hline
\end{tabular}

${ }^{*}$. This is a lower bound of the true significance.

a. Lilliefors Significance Correction

Fonte: Pesquisa de campo

Como a amostra para esta pesquisa é menor do que 50 casos (17 casos), o modelo a ser utilizado para verificação de aderência à curva normal é o de Shapiro-Wilk, onde:

H0: A distribuição é normal.

H1: A distribuição não é normal.

(nível de significância) $=0,05$

Se Sig. (p) < 0,05 Rejeita-se H0.

Se Sig. (p) > 0,05 Aceita-se H0.

No caso das variáveis apresentadas, somente a Afinidade apresenta valor superior a 0,05 , ou seja, somente esta variável possui aderência à curva normal. Portanto, utiliza-se análise de correlação não paramétrica de Spearman, como mostrado no Quadro 2:

Quadro 2 - Teste de Correlação entre as Variáveis Ordinais Correlations

\begin{tabular}{|c|c|c|c|c|c|c|c|}
\hline & & & $\begin{array}{l}\text { Afinidade - } \\
\text { Dicotomizada } \\
\text { (Indegree) }\end{array}$ & $\begin{array}{l}\text { Disposição } \\
\text { em Colaborar }\end{array}$ & Idade & $\begin{array}{l}\text { Tempo de } \\
\text { Empresa } \\
\text { em Meses } \\
\end{array}$ & $\begin{array}{l}\text { Salário } \\
\text { Mensal } \\
\end{array}$ \\
\hline \multirow[t]{15}{*}{ Spearman's rho } & \multirow{3}{*}{$\begin{array}{l}\text { Afinidade-Dicotomizada } \\
\text { (Indegree) }\end{array}$} & Correlation Coefficient & 1,000 & $.744^{\star x}$ & .327 & $.567^{*}$ & $603^{\star}$ \\
\hline & & Sig. (2-tailed) & . &, 001 & 201 & .018 &, 010 \\
\hline & & $N$ & 17 & 17 & 17 & 17 & 17 \\
\hline & \multirow[t]{3}{*}{ Disposição em Colaborar } & Correlation Coefficient &, $744^{* *}$ & 1,000 &, 311 &, 454 & $567^{\star}$ \\
\hline & & Sig. (2-tailed) &, 001 & . & 224 & .067 &, 018 \\
\hline & & $\mathrm{N}$ & 17 & 17 & 17 & 17 & 17 \\
\hline & \multirow[t]{3}{*}{ Idade } & Correlation Coefficient & 327 & 311 & 1,000 & 347 &, 354 \\
\hline & & Sig. (2-tailed) & 201 & .224 & & .173 &, 164 \\
\hline & & $\mathrm{N}$ & 17 & 17 & 17 & 17 & 17 \\
\hline & \multirow{3}{*}{$\begin{array}{l}\text { Tempo de Empresa em } \\
\text { Meses }\end{array}$} & Correlation Coefficient & $.567^{\star}$ & .454 & .347 & 1,000 &, 365 \\
\hline & & Sig. (2-tailed) &, 018 &, 067 &, 173 & . &, 150 \\
\hline & & $\mathrm{N}$ & 17 & 17 & 17 & 17 & 17 \\
\hline & \multirow[t]{3}{*}{ Salário Mensal } & Correlation Coefficient & $603^{*}$ & $567^{\star}$ &, 354 & 365 & 1,000 \\
\hline & & Sig. (2-tailed) &, 010 &, 018 &, 164 &, 150 & . \\
\hline & & $N$ & 17 & 17 & 17 & 17 & 17 \\
\hline
\end{tabular}

${ }^{* *}$. Correlation is significant at the 0.01 level (2-tailed).

${ }^{*}$. Correlation is significant at the 0.05 level (2-tailed).

Fonte: Pesquisa de campo 
H0: Não existe correlação entre as variáveis.

H1: Existe correlação entre as variáveis.

(nível de significância) $=0,05$

Se Sig. (p) < Rejeita-se H0.

Se Sig. (p) > Aceita-se H0.

Através dos dados de correlação gerados pelo SPSS e demonstrados no Quadro 2, nota-se que as variáveis Afinidade e Salário possuem correlação com a variável Disposição em Colaborar, pois somente estas possuem significância menor que 0,05 .

A variável Setor é uma variável nominal, portanto não utiliza-se o teste de Spearman para análise de correlação com a variável Disposição em Colaborar. Neste caso, a opção foi o teste Cross-Tab / Chi-Quadrado, conforme demonstrado no Quadro 3.

Quadro 3 - Teste Cross-Tab / Chi-Quadrado entre Setor e Disposição em Colaborar Setor de Trabalho * Disposição em Colaborar Crosstabulation

\begin{tabular}{|c|c|c|c|c|c|c|c|c|c|c|c|}
\hline & & \multicolumn{9}{|c|}{ Disposição em Colaborar } & \multirow[b]{2}{*}{ Total } \\
\hline & & 4.0 & 5,0 & 6,0 & 7,0 & \begin{tabular}{l|l}
7,5 \\
\end{tabular} & 8,0 & 8,5 & 9,0 & 9,5 & \\
\hline \multirow{6}{*}{$\begin{array}{l}\text { Setor de } \\
\text { Trabalho }\end{array}$} & BANCADA & 2 & 1 & 1 & 0 & 0 & 1 & $\overline{0}$ & 0 & 0 & 5 \\
\hline & $\mathrm{CNC}$ & 0 & 0 & 0 & 1 & 0 & 1 & 0 & 2 & 0 & 4 \\
\hline & EXPEDIÇÃO & 0 & 0 & 0 & 0 & 0 & 0 & 0 & 1 & 1 & 2 \\
\hline & FRESA & 0 & 0 & 0 & 1 & 0 & 1 & 0 & 0 & 0 & 2 \\
\hline & SERRA & 0 & 0 & 0 & 0 & 0 & 1 & 0 & 0 & 0 & 1 \\
\hline & TORNO & 0 & 0 & 0 & 0 & 1 & 1 & 1 & 0 & 0 & 3 \\
\hline Total & & 2 & 1 & 1 & 2 & 1 & 5 & 1 & 3 & 1 & 17 \\
\hline
\end{tabular}

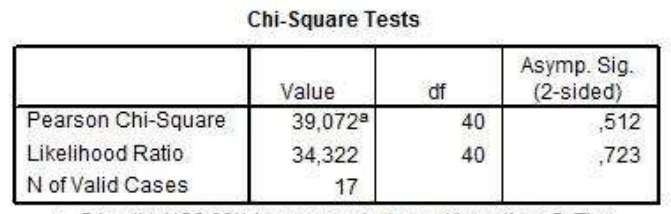

a. 54 cells $(100,0 \%)$ have expected count less than 5 . The minimum expected count is, 06 .

Fonte: Pesquisa de campo

H0: Não há diferença na Disposição em Colaborar entre os setores.

H1: Há diferença na Disposição em Colaborar entre os setores.

(nível de significância) $=0,05$

Se Sig. (p) < Rejeita-se H0.

Se Sig. (p) > Aceita-se H0.

Através dos dados gerados pelo SPSS e demonstrados no Quadro 3, onde a significância foi igual a 0,512, verifica-se que não há diferença na Disposição em Colaborar entre os setores, ou seja, a Disposição em Colaborar de um funcionário independe do setor onde ele trabalha. 


\section{Conclusão}

Como resultado da pesquisa, pode-se afirmar que as duas variáveis que possuem correlação com a disposição em colaborar são salário e afinidade, onde a variável afinidade apresentou grau de correlação superior ao salário, confirmando assim que o grau de inserção de um indivíduo na rede de relações da empresa está diretamente relacionado à disposição em colaborar deste. Dessa forma, a relação social em rede constitui o suporte para a disposição e colaboração dos processos, quer como forma de organização das informações, quer como meio de desenvolvimento das redes colaborativas de produção e conhecimento.

A colaboração, na dimensão social, tem um papel fundamental no desenvolvimento das interações que conduzem às diferentes formas de participação e criação de conhecimento, a partir da rede de relações entre os membros. Assim, o desenvolvimento da disposição em colaborar entre os funcionários é de interesse dos gestores. No ambiente de uma pequena empresa, como o deste estudo, onde os procedimentos escritos são normalmente raros ou até inexistentes e a transferência de conhecimento é, na sua maioria, feita através de conhecimento tácito, onde o fomento da rede social da empresa passa a ser de grande importância, resultando em aumento da disposição em colaborar dos funcionários.

Mesmo identificando isso, muitas vezes os gestores têm dificuldades de identificar maneiras de estimular a cooperação dos funcionários entre eles e com a empresa. De fato ainda existem alguns gestores que acreditam que a melhoria salarial é a única forma para isso, e por isso acreditam que essa melhoria, muitas vezes, não está ao alcance deles. Seja por não possuírem tal poder de decisão ou pela realidade de que a saúde financeira da empresa não esteja boa o suficiente para isso.

Com o resultado da análise dos dados desse artigo, identificou-se que se o gestor cria formas de incrementar a inclusão dos funcionários na rede social da empresa, principalmente aqueles que foram identificados como isolados, provavelmente haverá aumento nos índices de disposição em colaborar dos mesmos. As atividades sociais como festas de fim de ano e outras confraternizações podem ser uma boa alternativa para esse incremento da densidade da rede social da empresa.

A importância desse artigo é que se trata de um tema com possibilidades de reaplicação em outras empresas ou departamentos, onde poderá ser verificado se há a existência desta correlação entre a variável afinidade e a variável disposição em colaborar. Poderá também, se comprovada essa correlação, possibilitar ao gestor trabalhar para o incremento da inclusão dos funcionários na rede social da empresa, onde o resultado desse trabalho provavelmente será o aumento da cooperação. Essa maior cooperação pode se transformar em aumento de produtividade, pois o trabalho em equipe será mais eficaz e, assim, o aumento da competitividade da organização. 
Considerando a possibilidade de que a análise e conclusões desta pesquisa sejam significativas, as relações sociais advindas de uma rede podem constituir um instrumento e/ou modelo organizacional. Assim, essa análise pode ser utilizada não só para a promoção dos processos participativos das atividades em grupo, mas também como meio para a elaboração de estratégias que minimizem a distância da rede social entre os componentes, podendo dessa forma aumentar a participação organizacional e produtiva através do aumento da disposição em colaborar dos funcionários.

É importante destacar que existem limitações quanto à técnica utilizada de Análise de Redes Sociais (SNA - Social Network Analysis), pois o levantamento dos dados através da aplicação deste tipo de instrumento possui interpretação subjetiva, assim o nível de exatidão das respostas pode depender de como o entrevistado interpretou e avaliou a escala de valores. Outra limitação é que o teste foi aplicado em apenas uma empresa, e seria de grande valor a reaplicação deste em outras empresas para que se possa ter uma confirmação dessa correlação com um universo de pesquisa maior.

\section{Abstract}

The main objective of this inquiry was to identify if the individuals who receive higher indication of affinity from the members of the social net in which they are inserted in the enterprise are those considered "owners" of a bigger cooperative participation from the administrators. The study was carried out with 17 officials of the productive sector of a small metallurgical enterprise located in São Caetano do Sul - SP. Initially, there was used the Analysis of Social Nets technique (SNA Social Network Analysis) through the software UCINET 6.0, considering the affinity criteria. Each official indicated a level of affinity that each one had with many of the participants of the net. THE SNA allows several analyses of the relations between the useful component of the net for the administrators and some of them are shown in this article. However the main focus of the work was to identify firstly which individuals (knots of the net) receive a higher number of demonstrations of affinity (variable affinity). In a second stage there was collected the perception of the administrators of the sector regarding the arrangement in collaborating of each official (variable arrangement in collaborating). Finally, using the software SPSS 15 statistical test took place where the existence of strong correlation was checked between the two variables. The results indicate that if the administrators look for new forms of stimulating the social insertion of workers probably the participation level of cooperation there will increase.

Key-Words: social networks, collaborative behavior, affinity.

\section{Referências}

BENGHOZI, P. J. Les Communautés Virtuelles: Structuration Sociale ou Outil de Gestion? Entreprises et Histoire. n $43 ;$ p.67-81. France, 2006.

BROWN, J. S.; DUGUID, P. The Social Life of Information. Harvard Business School Press. Boston, Massachusetts, 2002.

CASTElls, M. A. Sociedade em Rede. Paz e Terra. 2 ed. Rio de Janeiro, 2003. 
CROSS, R.; NOHRIA, N.; PARKER, A. Six Myths about Informal Networks - and How to Overcome Them. Mit Sloan Management Review, v.43, n..3, 2002.

FIGUEIREDO, D. A.; AFONSO, A. P. Managing Learning in Virtual Settings: The Role of Context. Information Science Publishing, Idea Group Inc. USA, 2006.

FLEMING, L.; MARX, M. Managing Innovation in Small Worlds. Mit Sloan Management Review, v. 48; n. 1; USA, 2006.

GIL, A.C. Como Elaborar um Projeto de Pesquisa. 4 ed. São Paulo: Atlas, 2006.

GUIMARÃES, F. J. Z. M.; SOUSA, E. Diagnóstico Utilizando Análise de Redes Sociais [Rio de Janeiro] 2005 VIII, 51 p. 29,7 cm (COPPE/UFRJ, Especialização, Engenharia de Produção), 2005.

HATALA, J. P. Social Analysis in Human Resource Development: A New Methodology. Human Resource Development Review, v. 15, 1, p. 49-71, 2006.

cross ref

HUYSMAN, M.; WULF, V. It to Support Knowledge Sharing in Communities, Towards a Social Capital Analysis. Journal of Information Technology. n 21, p. 40-51, USA, 2006.

LEA et al. Enhancing Business Networks Using Social Network Based Virtual Communities. Industrial Management Data Systems. 106, p 121-138. ABI/INFORM Global, 2006.

MARTELETO, R. M. Analysis of Social Networks - Application in the Studies of Information Transfer. Ciência da Informação. Jan/Apr., v.30, n.1, 2001.

MORENO, J.L. Who shall survive? Washington, DC: Nervous and Mental Disease, 1934.

RIZOVA, P. Are You Network for Successful Innovation? Mit Sloan Management Review. Sprin, v. 1 47, n3. p. 49$56,2002$.

STAHL, G. Analyzing and Designing the Group Cognitive Experience. International Journal of Cooperative Information Systems, 2006.

cross ref

WENGER, E. C.; SNYDER, W. M. Comunidades de Prática. In: Harvard Business Review. Aprendizagem Organizacional. Rio de Janeiro: Campus. p. 9-26, 2001.

\section{Dados dos autores:}

Nome: Marcos Eduardo Zambanini

Filiação Institucional: Universidade Municipal de São Caetano do Sul

Departamento: PPGA da USCS

Endereço completo para correspondência: Estrada das Lágrimas, 3621 - Bloco 09 - Apto 133 - São

João Clímaco - São Paulo - SP - CEP 04244-000

Função ou cargo ocupado: Doutorando em Administração

Telefone para contato: (11) 9-8211-4140

e-mail: zambanini@uol.com.br 
Nome: Thais Ettinger Oliveira

Filiação Institucional: Universidade Municipal de São Caetano do Sul

Departamento: PPGA da USCS

Endereço completo para correspondência: Endereço residencial: Rua Deputado Laercio Corte, 230.

Apt 11, Paraíso do Morumbi. CEP 05706-290

Função ou cargo ocupado: Doutoranda em Administração

Telefone para contato: (11) 4239-3200

e-mail: thaisenttinger@hotmail.com

\section{Nome: Marco Antonio Pinheiro da Silveira}

Filiação institucional: Universidade Municipal de São Caetano do Sul

Departamento: PPGA da USCS

Endereço completo para correspondência: Rua Santo Antonio 50, Centro, São Caetano do Sul - SP09521-160

Função ou cargo ocupado: Professor.

Telefone para contato: (11) 4239-3200

e-mail: marco.pinheiro@uscs.edu.br

Enviado em: 19/03/2012

Aprovado em: 21/11/2012 